\title{
Spectral differences between the radio-loud and radio-quiet low-hard states of GRS 1915+105: Possible detection of synchrotron radiation in $\mathrm{X}$-rays
}

\begin{abstract}
S. V. Vadawale ${ }^{1}$, A. R. Rao ${ }^{1}$, and S. K. Chakrabarti ${ }^{2}$
1 Tata Institute of Fundamental Research, Homi Bhabha Road, Mumbai 400 005, India

2 S.N. Bose National Center for Basic Sciences, Salt Lake, Calcutta 700 091, India

Received 22 December 2000 / Accepted 28 March 2001

Abstract. The Galactic microquasar GRS 1915+105 exhibits several episodes of steady X-ray emission characterized by a hard power-law spectrum and intense Quasi Periodic Oscillations. It is known that there are two types of such low-hard states, one with steady radio emission and the other without any significant radio emission. We present the results of a detailed X-ray spectroscopic study of GRS 1915+105, using data from the Rossi X-ray Timing Explorer obtained during various episodes of the low-hard states of the source. We show that there are distinct X-ray spectral differences between the radio-quiet and radio-loud low-hard states of the source. The X-ray spectra of the radio-quiet low-hard state is best described by a model consisting of a multicolor disk-blackbody and a Comptonized component, whereas the X-ray spectra of radio-loud low-hard state requires a model consisting of three components: a multicolor disk-blackbody, a Comptonized component and a power-law, for statistically and physically acceptable fits. We attempt to model the presence of this additional power-law component as due to synchrotron radiation which is responsible for the radio and infrared radiation from the source. We show that a simple adiabatically expanding jet model for the synchrotron radiation can account for the observed X-ray flux for reasonable values of the magnetic field and the mass outflow rate. This is the first report of detection of the synchrotron radiation in the X-ray band for this source.
\end{abstract}

Key words. accretion, accretion disks - black hole physics - stars: winds, outflows - stars: individual: GRS1915+105 - X-rays: stars

\section{Introduction}

The X-ray transient source GRS 1915+105 was discovered in 1992 (Castro-Tirado et al. 1992) and it earned the name microquasar due to the detection of radio lobes moving at apparent superluminal velocities from it (see Mirabel \& Rodriguez 1999 and references therein). It has been in an $\mathrm{X}$-ray bright state ever since its discovery and it shows various types of X-ray variability characteristics (Chen et al. 1997; Morgan et al. 1997; Muno et al. 1999; Yadav et al. 1999; Belloni et al. 2000; Rao et al. 2000).

Belloni et al. (2000) found that all the X-ray variability characteristics of GRS $1915+105$ can be understood as transition from three basic states: state A and B characterized by a soft spectrum and state $\mathrm{C}$ characterized by a hard power-law and intense $0.5-10.0 \mathrm{~Hz}$ QPO. The source can remain in the state $\mathrm{C}$ for long durations, which are defined as class $\chi$ (further subdivided into $\chi_{1}, \chi_{2}, \chi_{3}$ and $\chi_{4}$ ) by Belloni et al. (2000). Trudolyubov et al. (1999) studied the 1996/1997 low-hard state (class $\chi_{2}$ ) and the state

Send offprint requests to: S. V. Vadawale, e-mail: santoshv@tifr.res.in transitions and concluded that the QPO centroid frequency is correlated with the spectral and timing parameters and these properties are similar to other Galactic black hole candidates in the intermediate state. Rao et al. (2000) quantified the spectral states and found that state transitions can occur in a very short time in GRS $1915+105$.

The $\chi_{1}$ and $\chi_{3}$ classes have similar properties characterized by high $\mathrm{X}$-ray flux $(\sim 500 \mathrm{mCrab})$ and high radio flux ( $\sim 50$ mJy at $2.2 \mathrm{GHz}$ ), which can be associated with the radio-loud "plateau" state of the source (Fender et al. 1999). The $\chi_{2}$ and $\chi_{4}$ classes, on the other hand, have low $\mathrm{X}$-ray flux ( $\sim 250 \mathrm{mCrab})$ and low radio flux (<20 mJy). Recently, Rao et al. (2000) identified three X-ray spectral components during a $\chi_{3}$ state of the source and found that one of the components (the thermal Compton component) is responsible for the QPOs. Here we present a detailed X-ray spectroscopic study of the source during the steady low-hard states (the $\chi$ class) of the source using data from the RXTE archives. We divide the class $\chi$ into two generic sub-classes $\chi_{\mathrm{RL}}$ (radio-loud) and $\chi_{\mathrm{RQ}}$ (radio-quiet) according to the observed accompanying 


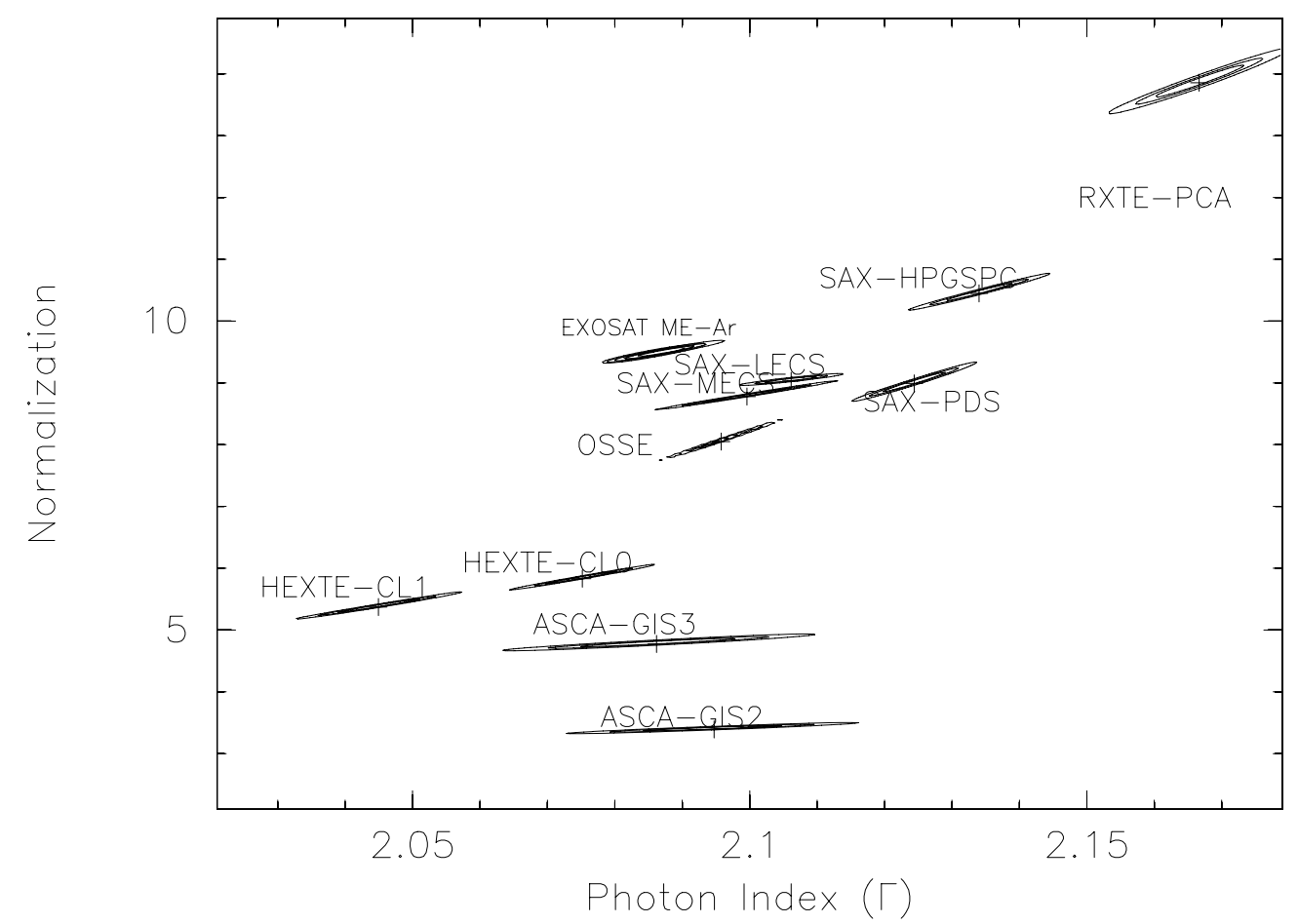

Fig. 1. Confidence contours for the normalization and the photon-index for the Crab Nebula (and pulsar) in the $0.5-180 \mathrm{keV}$ range obtained from various X-ray missions.

radio emission (Fender et al. 1999). The sub-class $\chi_{\mathrm{RL}}$ includes $\chi_{1}$ and $\chi_{3}$ and the sub-class $\chi_{\mathrm{RQ}}$ includes $\chi_{2}$ and $\chi_{4}$ classes. We find a distinct $\mathrm{X}$-ray spectral difference between the radio-loud and radio-quiet states of the source and we attempt to identify one of the X-ray spectral components with the synchrotron emission from the source. If confirmed, this will be the first detection of X-ray synchrotron emission from this source.

\section{Analysis and results}

\subsection{Characterization of instruments}

Rossi X-ray Timing Explorer (RXTE) was launched by NASA on 1995 December 31, with the main objective of timing studies of celestial X-ray sources. It has made great contributions to our understanding of High Energy Astrophysics by means of its unrivaled timing resolution. However, here we attempt to utilize the excellent opportunity of wide band hard X-ray spectroscopy, provided by the two narrow field of view instruments on board the RXTE: PCA (Jahoda et al. 1996) and HEXTE (Rothschild et al. 1998). In order to understand the systematics of the two instruments from the end user's perspective, we first analyzed the RXTE archival data of the standard candle source, the Crab. We have also analyzed the time averaged Crab data (pulsar and nebula taken together) from other missions like BeppoSAX, ASCA and EXOSAT. Results of our multi-mission study are shown in Fig. 1 (for a detailed discussion on these results see Rao \& Vadawale 2000). We find that the wide band $(0.5-180 \mathrm{keV})$ Crab spectra can be described by a single power-law with a photon index of 2.1, for most of the instruments. However, two instruments, HEXTE Cluster 1 and PCA, show systematic deviation from this value. HEXTE Cluster 1 gives a low photon index. It needs some additional systematic errors to give an acceptable fit and also one of the four detectors in this cluster is not capable of giving spectral data. Therefore we use data only from HEXTE Cluster 0 in our analysis.

The PCA on the other hand gives a high photon index. The problem with PCA is a little more than just higher photon index. Figure 2 shows the residual for a fit to the Crab data with the photon index frozen at 2.1. The existence of a huge excess below $10 \mathrm{keV}$ shows that there might be some systematic feature in the instrument which is not properly understood. Trying to fit the data with a single power-law gives an unacceptable fit $\left(\chi_{\nu}^{2} \approx 50\right)$ with a higher photon index and large " $\mathrm{S}$ " shape residuals below $10 \mathrm{keV}$. The spectral fit can be made acceptable by adding a $2 \%$ systematic error, however the photon index is higher (this was also noted by Gierlinski et al. 1999) and some residuals below $10 \mathrm{keV}$ still appears. Because of this soft excess, any feature below $10 \mathrm{keV}$ should be interpreted with caution. Therefore, since we wish to attempt wide band spectroscopy, in general we avoid adding components like line or edge which have only a local effect and requires high spectral resolution, in the spectral analysis with PCA. We find that an exponential modification of the form $N(E)=1+A \mathrm{e}^{-f E}$ (where $A$ is amplitude and $f$ is a constant and $E$ is energy in $\mathrm{keV}$ - model expfac in XSPEC), at low energies to the power-law with photon index 2.1 can explain the Crab data from PCA. Parameters 


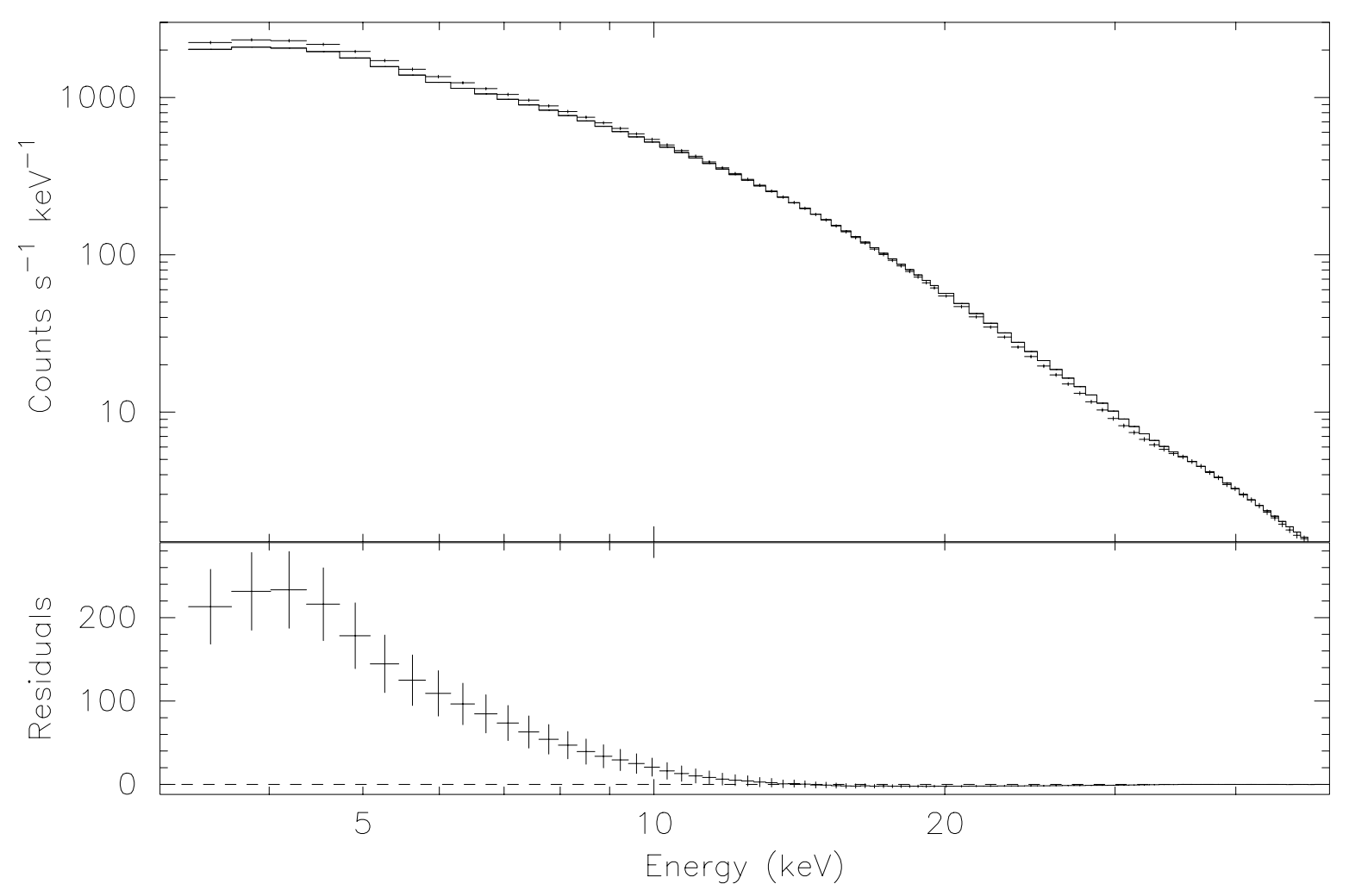

Fig. 2. A single power-law fit to the Crab data from RXTE-PCA with photon index fixed at value 2.1. The huge and systematic residuals below $10 \mathrm{keV}$ shows that there is some detector feature which is not properly understood.

Table 1. Spectral fits to the Crab data from individual PCUs from RXTE-PCA with a model consisting of a power-law, exponential modification to the power-law at lower energies and absorption by interstellar medium ${ }^{1}$.

\begin{tabular}{lcccc}
\hline \hline Detector & $N_{\text {pow }}$ & $A$ & $f$ & $\chi^{2}$ (dof) \\
\hline \hline PCU 0 & $9.27 \pm 0.12$ & $0.331 \pm 0.035$ & $0.114 \pm 0.020$ & $63.33(81)$ \\
PCU 1 & $9.53 \pm 0.12$ & $0.288 \pm 0.032$ & $0.109 \pm 0.021$ & $83.34(83)$ \\
PCU 2 & $9.79 \pm 0.13$ & $0.383 \pm 0.030$ & $0.099 \pm 0.015$ & $97.35(81)$ \\
PCU 3 & $10.50 \pm 0.15$ & $0.308 \pm 0.026$ & $0.089 \pm 0.016$ & $102.50(84)$ \\
PCU 4 & $10.45 \pm 0.13$ & $0.320 \pm 0.036$ & $0.125 \pm 0.021$ & $77.23(78)$ \\
\hline
\end{tabular}

${ }^{1} N_{H}$ is frozen at $0.4 \times 10^{22} \mathrm{~cm}^{-2}$ which is the average value obtained from a fit to data from low energy detectors of ROSAT, BeppoSAX and ASCA. Values of the photon-index and start energy for the exponential modification are frozen at 2.1 and $0.5 \mathrm{keV}$ respectively.

of this model can be used to quantify the soft excess. We have checked whether this soft excess can be attributed to any particular Proportional Counter Unit (PCU). Table 1 shows that this soft excess, of the same order, is present in all the PCUs and it is not possible to identify a few of them as good detectors. We have also verified that the results obtained from wide-band spectral fitting does not depend on selection of a combination of PCUs. Therefore in our wide band spectral analysis we use data from all PCUs added together with $2 \%$ systematic error along with the data from HEXTE Cluster-0.

\subsection{Data reduction and spectral fitting}

We have selected representative observations for each subclass $\chi_{\mathrm{RQ}}$ and $\chi_{\mathrm{RL}}$. Table 2 gives the details of these individual observations. The data reduction and analysis was performed with the software FTOOLS (Ver 5) and XSPEC (Ver 11). We extracted 129 channel spectra from PCA Standard-2 data (data from all PCUs added together) and 64 channel spectra from HEXTE Cluster-0 archive data. We generated the corresponding background spectra and response matrices following the standard procedures. We fitted the PCA (3-50 keV) and HEXTE-CL0 (15-180 keV) spectra simultaneously with different spectral models such as disk-blackbody; disk-blackbody and comptonization due to hot plasma (CompST - see Sunyaev \& Titarchuck 1980); disk-blackbody and cutoff power-law; disk-blackbody, CompST and power-law; etc. modified by interstellar absorption. We fixed the value of the interstellar absorption to $N_{H}=6 \times 10^{22} \mathrm{~cm}^{-2}$ (Belloni et al. 1997; 
Table 2. Results of spectral analysis of RXTE data of GRS 1915+105 during radio-loud and radio-quiet low-hard states.

A. Observation $\log$, radio flux ${ }^{1}$ and $\chi^{2}$ (dof) for different models ${ }^{2}$

\begin{tabular}{|c|c|c|c|c|c|c|c|}
\hline \multirow[t]{2}{*}{$\overline{\text { Date }}$} & \multirow[t]{2}{*}{ PID } & \multirow[t]{2}{*}{ Class } & \multirow{2}{*}{$\begin{array}{c}\text { Radio flux (mJy) } \\
\text { (at } 2.25 \mathrm{GHz})\end{array}$} & \multicolumn{4}{|c|}{$\chi^{2}$ (dof) } \\
\hline & & & & $\mathrm{dbb}+\mathrm{po}$ & $\mathrm{dbb}+\mathrm{ctpo}$ & $\mathrm{dbb}+\mathrm{co}$ & $\mathrm{dbb}+\mathrm{co}+\mathrm{po}$ \\
\hline 1997 Oct 16 & $20402-01-50-01$ & $\chi_{\mathrm{RL}}$ & 51.5 & $404.23(124)$ & $259.31(123)$ & $304.59(123)$ & $188.03(121)$ \\
\hline 1998 May 12 & $30402-01-12-01$ & $\chi_{\mathrm{RL}}$ & 68.6 & $366.46(124)$ & $233.94(123)$ & $276.21(123)$ & $171.42(121)$ \\
\hline 1999 Jun 7 & 40703-01-17-01 & $\chi_{\mathrm{RL}}$ & 31.4 & $221.85(115)$ & $176.87(114)$ & $249.45(114)$ & $140.42(112)$ \\
\hline 1996 Dec 4 & 20402-01-05-00 & $\chi_{\mathrm{RQ}}$ & 13.8 & $189.26(124)$ & $149.84(121)$ & $137.41(121)$ & - \\
\hline 1998 Aug 31 & $30703-01-31-00$ & $\chi_{\mathrm{RQ}}$ & 13.4 & 151.61(117) & $106.56(116)$ & $99.18(116)$ & - \\
\hline
\end{tabular}

B. Best fit X-ray spectral parameters ${ }^{3}$ and $2-50 \mathrm{keV}$ X-ray flux of individual spectral components

\begin{tabular}{|c|c|c|c|c|c|c|c|}
\hline \multirow[t]{2}{*}{ Date } & \multirow{2}{*}{$\begin{array}{c}k T_{\text {in }} \\
(\mathrm{keV})\end{array}$} & \multirow{2}{*}{$\begin{array}{c}k T_{\mathrm{e}} \\
(\mathrm{keV})\end{array}$} & \multirow[t]{2}{*}{$\tau$} & \multirow[t]{2}{*}{$\Gamma_{x}$} & \multicolumn{3}{|c|}{$2-50 \mathrm{keV}^{2}$ Flux $\left(10^{-9} \mathrm{erg} \mathrm{cm}^{-2} \mathrm{~s}^{-1}\right)$} \\
\hline & & & & & Disk Blackbody & CompST & Power-law \\
\hline 1997 Oct 16 & $1.99_{-0.04}^{+0.05}$ & $4.89_{-0.08}^{+0.09}$ & $10.25_{-0.27}^{+0.30}$ & $2.49_{-0.01}^{+0.01}$ & 4.65 & 7.78 & 9.41 \\
\hline 1998 May 12 & $1.73_{-0.03}^{+0.03}$ & $4.08_{-0.08}^{+0.08}$ & $12.60_{-0.59}^{+0.47}$ & $2.61_{-0.04}^{+0.04}$ & 7.93 & 5.50 & 11.33 \\
\hline 1999 Jun 7 & $1.52_{-0.10}^{+0.10}$ & $6.73_{-0.21}^{+0.23}$ & $5.82_{-0.16}^{+0.16}$ & $2.59_{-0.02}^{+0.02}$ & 1.96 & 12.87 & 9.33 \\
\hline 1996 Dec 4 & $1.28_{-0.36}^{+0.29}$ & $20.76_{-0.97}^{+1.11}$ & $2.94_{-0.10}^{+0.10}$ & - & 0.34 & 20.53 & - \\
\hline 1998 Aug 31 & $1.13_{-0.08}^{+0.09}$ & $21.96_{-2.19}^{+3.11}$ & $2.78_{-0.24}^{+0.21}$ & - & 2.23 & 19.13 & - \\
\hline
\end{tabular}

${ }^{1}$ Average flux for the respective day, from Green Bank Interferometer public data.

${ }^{2}$ The model components are dbb: disk-blackbody, po: power-law, ctpo: cutoff power-law, co: CompST.

${ }^{3}$ The spectral parameters are: $k T_{\text {in }}$ : Inner disk temperature $k T_{\mathrm{e}}$ : Electron temperature of the Compton cloud $\tau$ : Optical depth of the Compton cloud $\Gamma_{x}$ : Photon index.

Markwardt et al. 1999; Muno et al. 1999) and have used the normalization of HEXTE spectrum (with respect to the PCA spectrum) as a free parameter so as to account for any residual uncertainties in the relative area of the two instruments. For each observation, the values of $\chi^{2}$ (degrees of freedom - dof) for different models are given in Table 2A. The spectral parameters for the best fit model are given in Table $2 \mathrm{~B}$.

We find that addition of a Gaussian feature for iron $\mathrm{K}_{\alpha}$ line near $6.4 \mathrm{keV}$ in our models does reduce the $\chi^{2}$ values but does not have any effect on the spectral parameters of the other components. For example, for the data obtained on 1997 October 16, addition of a line feature reduces the value of $\chi_{\nu}^{2}$ to 1.2 (from 1.6), but the values of the parameters remain almost the same (within a few percent of the values). The derived value of the equivalent width is rather high $(\sim 150 \mathrm{eV})$. The high spectral resolution observation of this source using ASCA or Chandra show very low equivalent width $(10-20 \mathrm{eV})$ for iron $\mathrm{K}_{\alpha}$ line (Kotani et al. 2000; Lee et al. 2001). This suggests that the observed features might be mainly due to detector artifact. Further, the addition of line feature affects all the continuum models in almost identical way with similar reduction in the value of $\chi^{2}$. Since it does not affect our inference based on the wide band structure of the spectrum, we do not include the iron line in our fits.

\subsection{Radio-quiet low-hard state}

The standard model for the Black Hole Candidates (BHCs) consists of a multicolour disk-blackbody and a power-law. The power-law is generally assumed to be an approximation for some physical processes like Comptonisation of soft photons from a hot plasma. Since we have used wide band data upto $180 \mathrm{keV}$, we find that a power-law does not give a satisfactory fit to the X-ray spectra of the radio quiet low-hard state (Table 2), particularly because there are fewer counts at high energies $(>70 \mathrm{keV})$ than are predicted by the model. A model consisting of a disk-blackbody and a cutoff power-law gives a better fit, however, the best fit is obtained for a model consisting of a disk-blackbody and a CompST. The CompST appears to be the best candidate for the second component also due to the fact that it describes the actual process of the inverse compton scattering of the soft photons. The high energy cutoff also comes naturally in the CompST. Therefore we conclude that the $3-180 \mathrm{keV}$ spectra of the radio-quiet low-hard state is best described by the model consisting of a disk-blackbody and a CompST. It should be noted here that the wide band spectral fitting is critical to distinguish these models. It should also be pointed out here that the CompST model does not include relativistic corrections and hence more sophisticated models may be required if we include data above $200 \mathrm{keV}$.

\subsection{Radio-loud low-hard state}

The X-ray spectra of the radio-loud low-hard state is peculiar and needs a careful interpretation. Any combination of two spectral components gives a statistically inferior fit to the data. On examination of the residuals it is found that this is mainly due to the fact that the data has a spectral curvature in the $15-40 \mathrm{keV}$ region and a flattening above $70 \mathrm{keV}$, thus necessitating at least two spectral components above $15 \mathrm{keV}$ (or three component in the $3-180 \mathrm{keV}$ region). The improvement in the value of $\chi^{2}$ is more than 100 , thus conclusively showing that a combination at least three components are required to 


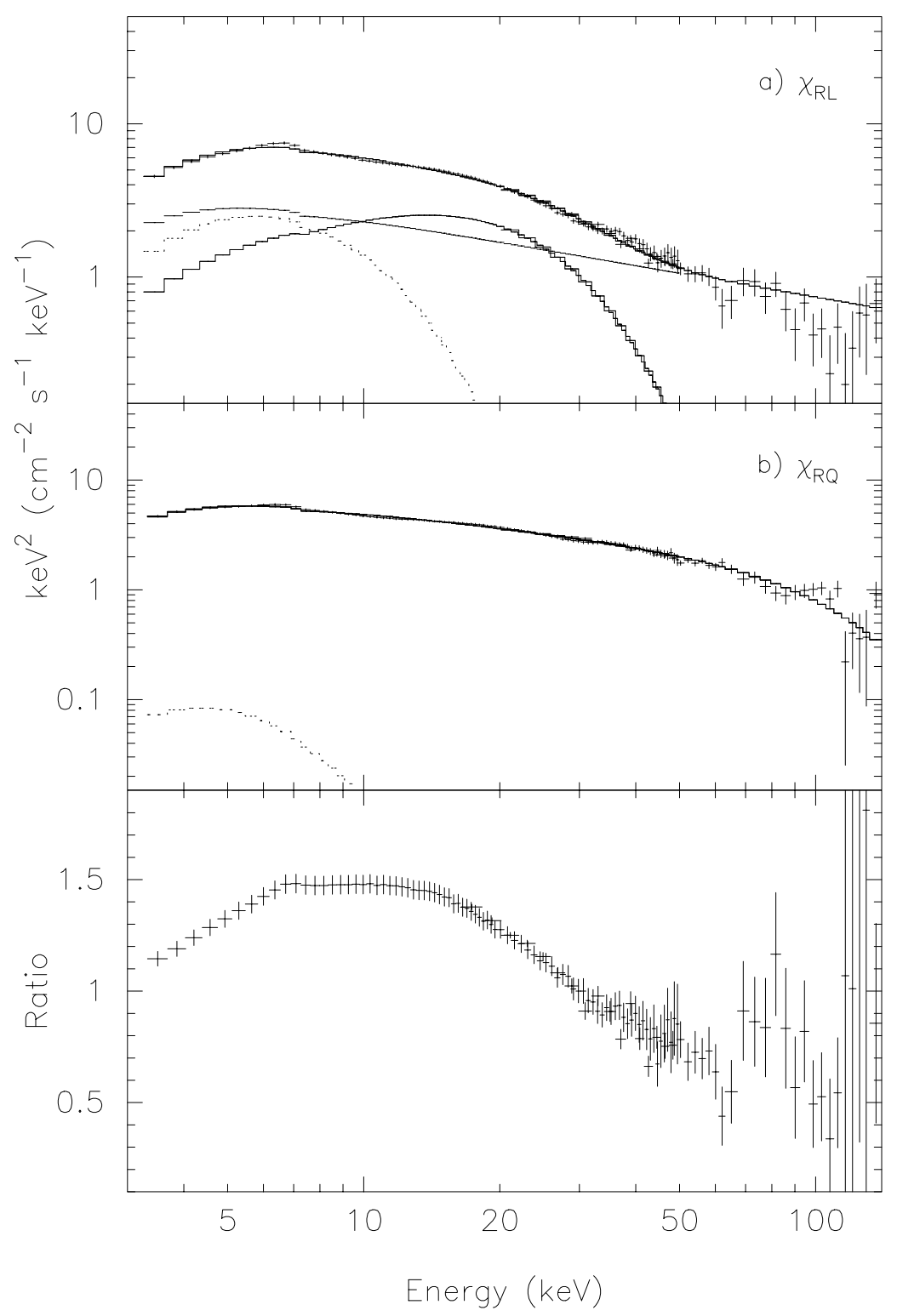

Fig. 3. The deconvolved spectra during the $\chi_{\mathrm{RQ}}$ and $\chi_{\mathrm{RL}}$ states of GRS $1915+105$ are shown in the top two panels of the figure. The model consists of a disk blackbody (shown as dotted lines) and emission from a Compton cloud. The $\chi_{\mathrm{RL}}$ state requires an additional power-law component. The bottom panel shows the ratio of observed count rates ( $\chi_{\mathrm{RL}}$ to $\chi_{\mathrm{RQ}}$ ratio), which highlights the spectral difference in the $15-50 \mathrm{keV}$ range between the two states.

adequately explain the wide band data of the radio-loud low-hard state of GRS $1915+105$.

This shows that the wide band X-ray spectrum of the radio-loud low-hard state is qualitatively different from that of the radio-quiet low-hard state. To highlight this difference we have shown in Fig. 3 the unfolded spectrum of the radio-loud low-hard state obtained on 1997 October 16 (top panel), radio-quiet low-hard state obtained on 1996 December 4 (middle panel) and the ratio of the observed count rates in the radio-loud state to those in the radio-quiet state in each energy channel (normalized to the value at $30 \mathrm{keV}$ ), in the bottom panel. The spectral curvature in the $15-45 \mathrm{keV}$ region can be seen even in the raw data.

In the fit with a disk-blackbody and a power-law, this spectral curvature in the $\chi_{\mathrm{RL}}$ class is mimicked by the disk-blackbody, giving an unrealistically high temperature $(4-5 \mathrm{keV})$. Further, the resultant values of $\chi^{2}$ are inferior to that obtained for the 3 component fit. Fit with only disk-blackbody and CompST is also not acceptable, now because CompST decreases much more sharply and there are more counts at higher energies $(>70 \mathrm{keV})$ than predicted. Thus an additional power-law is needed to account for them. It should be noted here that, though the existance of the three components is fairly certain here, the exact values of parameters of disk-blackbody and CompST should be used with caution, because of the large systematic error added to the PCA data.

The wide band X-ray spectra of GRS $1915+105$ in the radio-loud state has been reported earlier by Muno et al. (1999). This is the only reported X-ray spectra of radioloud low-hard state of GRS $1915+105$. They use a model 
consisting of disk blackbody, power-law and a gaussian line corresponding to iron $\mathrm{K}_{\alpha}$. We get similar spectral parameters when we use disk blackbody and power-law as the continuum models. As mentioned earlier, we get a better fit using the three component model. Further, the line equivalent width is quite high $(\sim 200 \mathrm{eV})$ compared to that observed with ASCA or Chandra. The derived parameters of the disk-blackbody (inner disk temperature $k T_{\mathrm{in}} \sim 4-5 \mathrm{keV}$ and normalization $\left.N_{\mathrm{bb}} \sim 0.5-0.7\right)$ appear to be quite unrealistic. Such a high inner disk temperature is not observed in any other $\mathrm{X}$-ray binary. The value of $N_{\mathrm{bb}}=0.7$ corresponds to an inner disk radius $R_{\mathrm{in}} \approx$ $1.8 \mathrm{~km}$ for this source $\left(R_{\mathrm{in}, \mathrm{km}}=D_{10 \mathrm{kpc}} \sqrt{N_{\mathrm{bb}} / \cos \theta}\right.$, $D=12.5 \mathrm{kpc}$ and $\theta=70)$. The inner disk radius of $1.8 \mathrm{~km}$ is clearly unphysical because the Schwarzschild radius for a one solar mass black hole is $\sim 3 \mathrm{~km}$.

\section{Origin of the additional power-law}

The previous section gives a consistent picture according to which, the disk-blackbody and CompST components are always present in the X-ray spectra of the low-hard state. During the low-hard state accompanied by high radio emission, the $\mathrm{X}$-ray spectra requires an additional power-law component. The $2-50 \mathrm{keV}$ X-ray flux of the additional power-law also shows some correlation with the accompanying radio flux (see Table $2 \mathrm{~B}$ ). This naturally leads to a hypothesis that the additional power-law component is also due to the same mechanism i.e. synchrotron radiation, which is responsible for the observed radio emission. Dhawan et al. (2000) have reported an AU scale radio jet from the core of the source during the $\chi_{3}$ state of the source observed in 1997 October. Recently Markoff et al. (2001) showed that the entire X-ray emission from a black hole binary XTE J1118+480 can be explained as synchrotron emission from the innermost part of a jet. Hence it is quite conceivable that the additional powerlaw component seen in X-rays is emitted from the base of such a jet.

\subsection{A synchrotron jet model}

Here we present a simplified model similar to that given by Hjellming \& Johnston (1988), to examine whether the power-law component can indeed be explained as a synchrotron emission from the base of a compact, adiabatically expanding conical jet in GRS $1915+105$. Since we are interested in the synchrotron radiation in X-rays, we consider emission from the entire volume of the jet instead of emission from only the outer layer of the jet as done by Hjellming \& Johnston (1988), who were mainly interested in the radio emission.

We make the following assumptions:

1. The mass outflow from regions close to the black-hole is conical in shape with a small opening angle $\theta\left(\sim 10^{\circ}\right)$;

2. Electron-proton plasma is injected at the base of the jet with relativistic velocities;
3. Electron acceleration occurs at the shock front located at distance $z_{\mathrm{s}}$ from the base, resulting in the power-law distribution in energy $N_{\mathrm{e}}=k E^{-p}$;

4. There is no mass loss across the sides of the jet. Magnetic field and particle distribution are uniform across any cross-section of the jet.

Let $B_{\mathrm{s}}$ and $k_{\mathrm{s}}$ be the magnetic field and proportionality constant of the power-law electron distribution at the shock front, and $r_{\mathrm{s}}$ be the radius of the cone at the shock front given by $r_{\mathrm{s}}=r_{0}+z_{\mathrm{s}} \tan (\theta / 2)$, where $r_{0}$ is the base radius of the jet assumed to be 100 Schwarzschild radius. Then from conservation of magnetic flux along the jet, we get (see Hjellming \& Johnston 1988)

$B_{\ell}=B_{\mathrm{s}}\left(\frac{r_{\ell}}{r_{\mathrm{s}}}\right)^{-1}$

where $r_{\ell}$ is the radius of the cone at a distance $\ell$ from the base, given by

$r_{\ell}=r_{0}+\ell \tan (\theta / 2)$.

Further, if we assume that the expansion with $r_{\ell}$ is adiabatic, then conservation of both the total energy and the number of relativistic particles requires that (see Hjellming \& Johnston 1988)

$k_{\ell}=k_{\mathrm{s}}\left(\frac{r_{\ell}}{r_{\mathrm{s}}}\right)^{-2(p+2) / 3}$.

The values of $B_{\ell}$ and $k_{\ell}$ determine the volume emissivity $J_{\ell}(\nu)$ and absorption coefficient $\chi_{\ell}(\nu)$ of the local medium (Longair 1994)

$$
\begin{aligned}
J_{\ell}(\nu)= & 2.344 \times 10^{-25} a(p) k_{\ell} B_{\ell}^{(p+1) / 2} \\
& \times\left(\frac{1.253 \times 10^{37}}{\nu}\right)^{(p-1) / 2} \mathrm{Wm}^{-3} \mathrm{~Hz}^{-1} \\
\chi_{\ell}(\nu)= & 3.354 \times 10^{-9} b(p) k_{\ell} B_{\ell}^{(p+2) / 2} \\
& \times\left(3.54 \times 10^{18}\right)^{p} \nu^{-(p+4) / 2}
\end{aligned}
$$

where $a(p)$ and $b(p)$, the functions of the the particle power-law index $p$ involving numerous $\Gamma$ functions, are of the order of unity. We consider the $\chi_{\mathrm{RL}}$ state observed during 1997 October, for which spectral index is $\sim 1.5$ (photon index $\sim 2.5$ ) which gives $p=4$. The values of $a(p)$ and $b(p)$, for $p=4$ are 1.186 and 0.230 (Longair 1994, Table 18.2).

Consider a segment of the cone with length $\mathrm{d} \ell$ at the distance $\ell$ from the base. This segment can be approximated by a cylinder of length $\mathrm{d} \ell$ and radius $r_{\ell}$. Now for this cylinder, consider a shell of thickness $\mathrm{d} r$ at the radius $r$ from the center. Total self absorbed synchrotron emission from this shell is (see Longair 1994)

$$
I_{\mathrm{S}}(\nu)=2 \pi r \mathrm{~d} \ell \frac{J_{\ell}(\nu)}{4 \pi \chi_{\ell}(\nu)}\left(1-\mathrm{e}^{-\chi_{\ell}(\nu) \mathrm{d} r}\right)
$$

where $2 \pi r \mathrm{~d} \ell$ is the area of the outer surface of the cylinder. This synchrotron radiation is absorbed in the medium of 
thickness $\left(r_{\ell}-r\right)$. Hence the observed spectrum of the radiation from this shell is

$I_{r}(\nu)=I_{\mathrm{s}}(\nu) \mathrm{e}^{-\chi_{\ell}(\nu)\left(r_{\ell}-r\right)}$.

Total observed spectrum from this segment is

$I_{\ell}(\nu)=\int_{0}^{r_{\ell}} I_{r}(\nu) \mathrm{d} r$

and the total emission spectrum from the entire conical jet is

$I(\nu)=\int_{0}^{L} I_{\ell}(\nu) \mathrm{d} \ell$

where $L$ is the assumed length of the jet. This is in the rest frame of the jet. To get the observed flux in the rest frame of the observer we have to transform it according to the transformations (Falcke \& Biermann 1996),

$\nu_{\mathrm{obs}}=D \nu, \quad I_{\mathrm{obs}}\left(\nu_{\mathrm{obs}}\right)=D^{2} I\left(\nu_{\mathrm{obs}} / D\right)$.

Where $D=[\gamma(1-\beta \cos \Theta)]^{-1}$ is the Doppler factor of the jet matter moving at the relativistic velocity $\beta c$ at angle $\Theta$ from the line of site. We assume them to be $0.9 \mathrm{c}$ and $70^{\circ}$ respectively.

Thus the observed flux is

$F(\nu)=\frac{D^{2} I(\nu) \sin \Theta}{4 \pi d_{\mathrm{sc}}^{2}}$

where $d_{\mathrm{sc}}$ is the distance to the source, which we assume to be $12 \mathrm{kpc}$ (Mirabel \& Rodriguez 1994).

We take a numerical approach to solve this radiation transfer problem. We divide the entire jet length in a large number of cylindrical segments $(\sim 100000)$, calculate the synchrotron emission from each segment assuming uniform $B_{\ell}$ and $k_{\ell}$ for each segment and finally get the observed flux by integrating over the jet length. The basic free parameters of this model are the magnetic field $B_{\mathrm{s}}$, the proportionality constant $k_{\mathrm{s}}$ at the shock front, the distance of the shock front from the base $z_{\mathrm{s}}$. The value $k_{\mathrm{s}}$ in itself is not physically meaningful, however it can be converted into a meaningful quantity like mass outflow rate. The total electron number density of the accelerated electrons, $N_{\mathrm{e}}$ is given by $k_{\ell} \int_{E_{\min }}^{E_{\max }} E^{-p} \mathrm{~d} E$. For $p>2$, it is governed by $E_{\min }$. Assuming that $50 \%$ of the electrons are accelerated at relativistic energies and that there is one proton per electron, mass density is given by $\rho=2 N_{\mathrm{e}} \gamma m_{\mathrm{p}}$. Then the total mass outflow rate is $\dot{M}_{\mathrm{o}}=\beta c \rho \pi^{2} R_{\mathrm{s}}$ (Falcke \& Biermann 1996). Thus, in order to get mass outflow rate $\dot{M}_{\mathrm{o}}$, the most important assumptions we have to make are about the lower energy cut-off of the electron energy distribution and velocity of the jet.

\subsection{Parameter space}

The predicted emission spectrum from the jet of length $100 \mathrm{AU}$ is shown in Fig. 4 (solid line) along with the observed points in X-ray as well as radio during the $\chi_{\mathrm{RL}}$ state of 1997 October. The parameters used in this calculation are $B_{\mathrm{s}}=5 \times 10^{3} \mathrm{G}, k_{\mathrm{s}}=1.2 \times 10^{-13}, z_{\mathrm{s}}=10^{5} R_{\mathrm{sw}}$ (Schwarzschild radius) and $\theta=10^{\circ}$. This value of $k_{\mathrm{s}}$ gives mass density $\rho_{\mathrm{s}}$ at the shock to be $\sim 3.7 \times 10^{-11} \mathrm{~kg} \mathrm{~m}^{-3}$, assuming lower energy cutoff $E_{\min }=1 \mathrm{GeV}\left(\gamma_{\min } \approx 2000\right)$ which requires the mass outflow rate of $\dot{M}_{\mathrm{o}}=2.3 \times$ $10^{15} \mathrm{~kg} \mathrm{~s}^{-1}$ for outflow velocity of $0.9 \mathrm{c}$. Total jet power given by these parameters is $4.28 \times 10^{39} \mathrm{erg} \mathrm{s}^{-1}$. Both the magnetic field $B_{\mathrm{s}}$ and $\dot{M}_{\mathrm{o}}$ are within the possible range (see next section). Taking $\gamma_{\min } \approx 100$ increases the required mass outflow rate by three order of magnitude.

As can be seen from Eq. (4), the same flux at any frequency can be obtained for various values of magnetic fields and electron densities i.e. mass outflow rates. The possible parameter space to get the observed X-ray flux is shown in Fig. 5. In this calculation we have assumed the values of many parameters, like velocity of the jet, jet angle, ratio of number density of the relativistic electrons to the total electron number density, base radius, distance of the source and angle between jet and line-of-sight. Among these, the parameters on which the mass outflow rate depends most sensitively are the velocity of the jet, ratio of number density of the relativistic electrons to the total electron number density, and the base radius. The values of distance of the source and the inclination angle are well known from the literature. Also, the final result does not depend on these parameters very sensitively. Among the remaining three, the assumption about the jet velocity is on the safer side, however, for the other two parameters we have assumed values which seem intuitively reasonable. It should be pointed out here that not all shown combinations of magnetic field and mass outflow rates produce radio fluxes similar to the observed flux because the turnover frequency is slightly different for different combinations. Also the turnover frequency strongly depends on the electron energy index, $p$. The observed radio data suggests a turnover frequency in the range $10^{14}-10^{15} \mathrm{~Hz}$. For physically reasonable magnetic field and mass outflow rates, this turnover frequency range can be obtained only for a narrow range of electron energy index around 4.0. Thus, apart from the observed X-ray power-law, the observed radio flux also requires a high electron energy index.

\section{Discussion}

Synchrotron emission from a conical jet was first studied by Blandford \& Konigl (1979) in the context of AGNs. Reynolds (1982) explored in more detail the observed spectra form winds and jets with a variety of geometries, magnetic fields and energetics. Hjellming \& Johnston (1988) discussed the application of such models to Xray binaries. Extending this further, Falcke \& Biermann (1996, 1999) developed a disk-jet "symbiosis" model to explain the radio emission from various accretion powered sources, according to which some fixed fraction of the accreted mass comes out as a jet. However, these models are developed mostly to account for the observed radio emission and does not discuss synchrotron emission in the high 


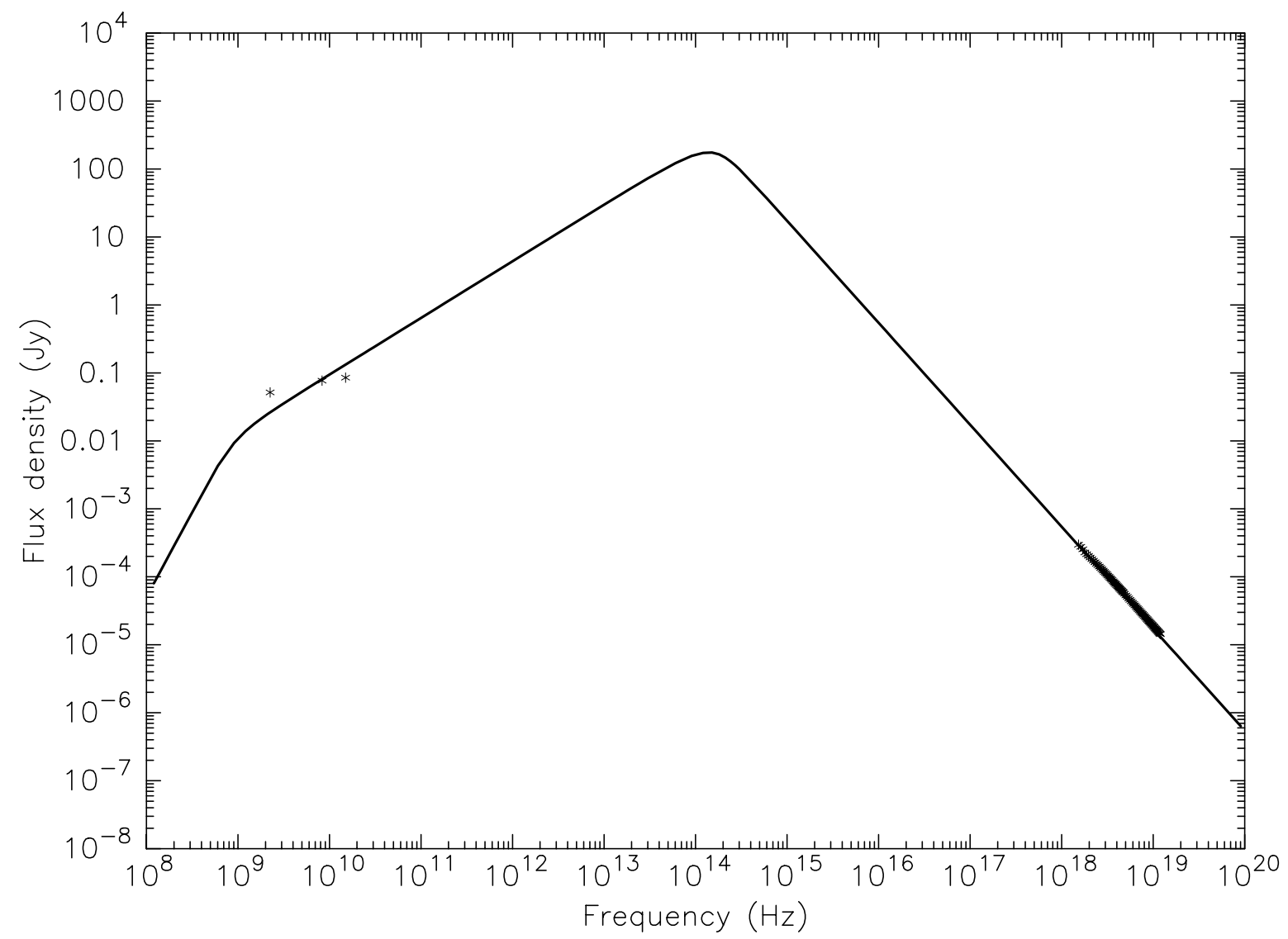

Fig. 4. A simplified model spectrum of synchrotron emission from a conical jet of length 100 AU with a magnetic field of $5 \times 10^{3} \mathrm{G}$ at the shock front. The stars represent observed fluxes on 1997 October 16, at $2.25 \mathrm{GHz}, 8.3 \mathrm{GHz}$ (GBI public data), $15 \mathrm{GHz}$ (Fender et al. 1999) and 20-150 keV (present work).

frequency range. Recently, Markoff et al. (2001) showed that, in a black hole binary, synchrotron emission can give observable X-ray flux. They showed that the entire X-ray flux of the black hole binary XTE J1118+480 can be explained as synchrotron emission. For XTE J1118+480 almost all the emission from the accretion disk is in the EUV range. In GRS 1915+105, however, the X-ray flux has significant contributions from the accretion disk as well as the inner Compton could. We have separated the flux from the additional power-law component and have attempted to identify it with the synchrotron emission and correlate with the observed radio emission.

It can be seen from Fig. 4 that the observed X-ray power-law agrees very well with that predicted by this simple synchrotron model, though the parameter space shown in Fig. 5 is a bit on extreme side. For the $\chi_{\text {RL }}$ state under consideration (1997 October), the inner disk temperature $\left(T_{\text {in }}\right)$ of $2 \mathrm{keV}$ gives a mass accretion rate of $\dot{M}_{\text {in }} \sim 10^{16} \mathrm{~kg} \mathrm{~s}^{-1}$. If we assume the outflow rate to be $\sim 10 \%$ of $\dot{M}_{\text {in }}$ (such outflows from very close to the compact object have been predicted by Chakrabarti 1999 and Das \& Chakrabarti 1999), then the minimum required magnetic field strength at the shock front is $\sim 10^{4} \mathrm{G}$. If we extend the same dependence back to the base of the jet, then the base magnetic field required is $\sim 10^{6} \mathrm{G}$. The base magnetic field value of the order of $10^{6} \mathrm{G}$ looks a bit higher, however, it should be noted that the magnetic field values of the order of $0.1 \mathrm{G}$, reported in the literature (Dhawan et al. 2000; Fender et al. 1999) are at a very large distance from the base and lead to a similar value if extrapolated back to the base. Markoff et al. (2001) have also reported that a magnetic field $\sim 10^{6}$ is required to explain the X-ray emission as synchrotron radiation, in the black hole binary XTE J1118+480. The scaling laws given by Narayan et al. (1998) also predict a similar value for the equi-partition magnetic field. This means that the base magnetic field values from $10^{5}$ to $10^{7} \mathrm{G}$ and corresponding mass outflow can produce the observed X-ray flux. The location of the shock, $z_{\mathrm{s}}$, also appears to be large, however, such a distance is required from the consideration of electron life-time in high $B$. Figure 5 shows that as $z_{\mathrm{s}}$ decreases the required $B_{\mathrm{s}}$ increases for a given $\dot{M}_{\mathrm{o}}$. Now a base magnetic field $B_{0}$ of the order of $10^{5}-10^{6}$ is expected, as discussed above. This makes $B_{\mathrm{s}}$ very large for smaller $z_{\mathrm{s}}$. The energy loss rate of electrons of energy $E$ in magnetic field $B$ is given by $-(\mathrm{d} E / \mathrm{d} t)=1.058 \times B^{2} \gamma^{2}(v / c)^{2} \mathrm{~W}$. For $B \sim 10^{5}$ this gives the life-time of an electron to be less than 0.1 second for any electron energy. Thus in such a strong magnetic field all electrons would thermalise immediately, which is not the case for this source, for which the radio emission is observed at $10 \mathrm{AU}$ (Dhawan et al. 2000). This suggests 


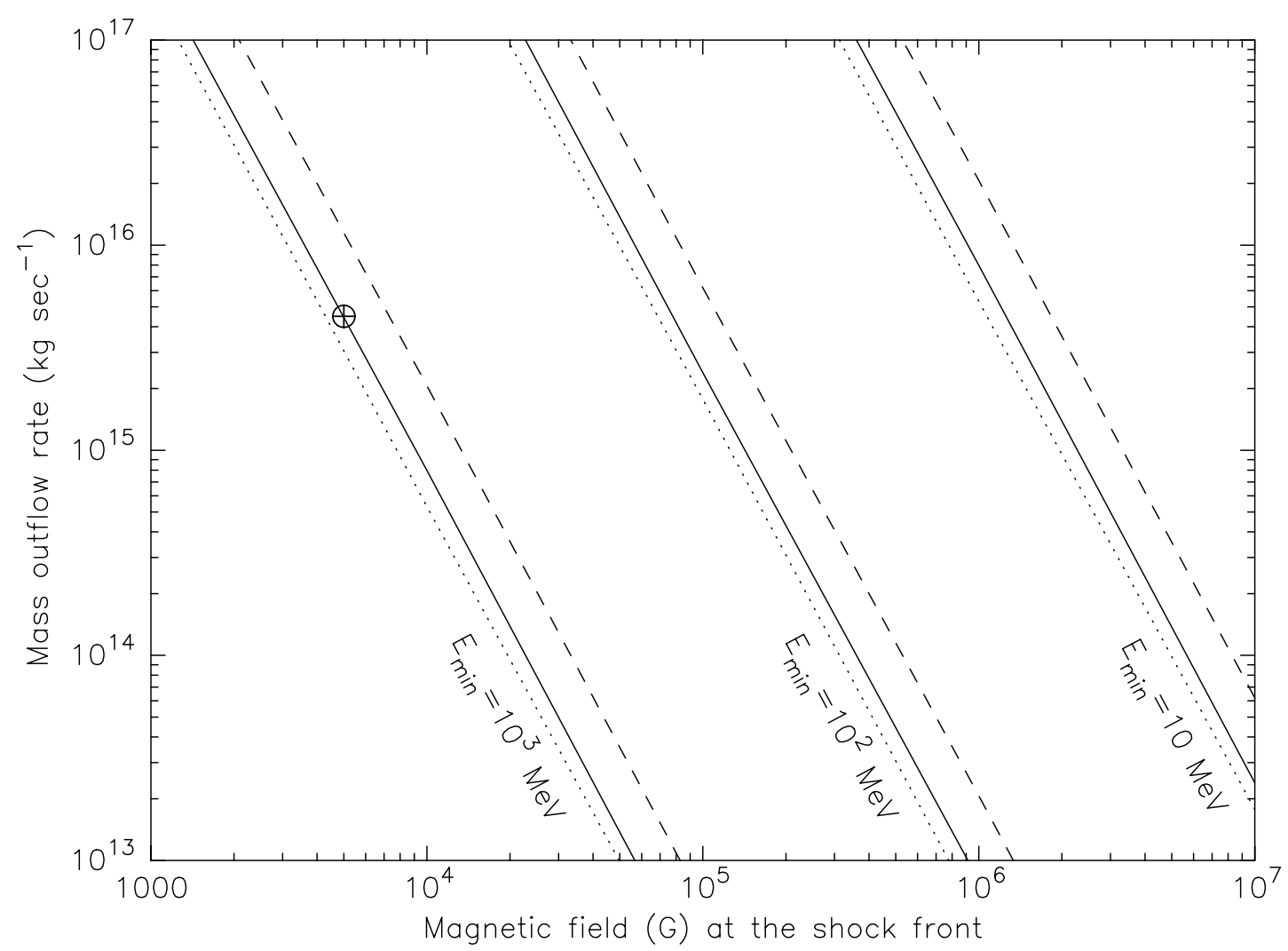

Fig. 5. Mass outflow rate vs. magnetic field at the shock front required to give the observed X-ray flux. The dashed lines represent the distance of the shock front $z_{\mathrm{s}}=5 \times 10^{4} R_{\mathrm{SW}}$, the solid lines represent $z_{\mathrm{s}}=10^{5} R_{\mathrm{SW}}$ and the dotted lines represent $z_{\mathrm{s}}=1.5 \times 10^{5} R_{\mathrm{SW}}$. Three groups of this lines are shown for different lower energy cutoff to the electron energy distribution. The circle shows the parameters used in the calculation of spectrum of Fig. 4.

that the electron acceleration site must be far away from the base where the magnetic field is moderately strong.

Figure 4 also shows that this model can predict the radio flux similar to that of the observed flux, however, the predicted spectral index in this frequency range is steeper than the observed value. According to this model, the spectral index in the optically thick region depends on the index of the electron energy distribution and is given by $\alpha_{\text {thick }}=10(p-1) /(7 p+8)$ (see Hjellming \& Johnston 1988 for derivation), which for present case $(p=4)$, is $\alpha_{\text {thick }}=0.833$ whereas the observed spectral index is $\sim 0.3$ during this observation. There are other models (Falcke 1996; Falcke \& Biermann 1999) which predict a much flatter spectral index in the optically thick region and which are also independent of the electron energy index. However, these problems are only for the optically thick frequencies i.e. frequencies below the turnover frequency. For optically thin frequencies, this model agrees with the observed data and hence the observed additional powerlaw component in the $\chi_{\mathrm{RL}}$ states can indeed be due to the synchrotron radiation for a wide range of physically meaningful parameters.

Recently Naik \& Rao (2000) have shown that, of the 12 classes in GRS $1915+105$ as defined by Belloni et al. (2000), high radio emission is present only during three classes: $\chi, \beta$ and $\theta$. The source is in the low-hard state during a significant fraction of time for both the classes $\beta$ and $\theta$. We have verified that the spectral properties of the low-hard states during these states are also similar to the $\chi_{\mathrm{RL}}$ state reported here. Thus it appears that, whenever there is a significant radio emission from the source (particularly with a flat or an inverted spectrum), it manifests itself in the X-ray spectra as an additional power-law component.

One more striking difference between the X-ray spectra of these two states is the different shape of CompST. The temperature of the Compton cloud, responsible for the Comptonized component, is lower in the radio-loud state, whereas the optical depth is much larger. Such a large optical depth has been reported earlier for this source (Rao et al. 2000). We have verified that optical depth of the order of unity can be rejected for the radio-loud state with very high statistical confidence (change in $\chi^{2}$ of $>200$ ). We find that the spectral curvature at $15-40 \mathrm{keV}$ needs a large optical depth, if this curvature is due to a Comptonization model. It should be noted here that according to the TCAF model of Chakrabarti \& Titarchuk (1995), the optical depth of the post-shock region should be of the order of the accretion rate (normalized to the Eddington accretion rate) and hence should be less than 1 . The observations reported here pertain to the case of high radio emission and related to an accretion disc with very high mass out-flow rate. It is quite possible that the region responsible for the thermal-Compton emission is a 
geometry where material outflow, Compton cooling and Comptonization of the disk emission is taking place and the parameters derived by the simple thermal-Compton model could be an approximation for the emission from such a complex region. Das \& Chakrabarti (1999) have proposed a combined inflow/outflow model in which they show that the outflow rate depends on the inflow parameters. It would be interesting to investigate the relation between the different Compton cloud properties in the presence of outflow.

\section{Conclusions}

Here we have shown that the X-ray spectra during the radio-loud and radio-quiet low-hard states of the Galactic microquasar GRS $1915+105$ are different. The most striking difference is the presence of an additional powerlaw component. We have shown that this component can arise due to the synchrotron radiation, by comparing the observed X-ray flux to the numerically estimated synchrotron spectrum from accelerated electrons at the shock front in a simple adiabatically expanding jet. Considerably wide ranges of mass outflow rate and magnetic fields at the shock can produce the observed X-ray power-law flux. This model can also predict the observed radio flux, however, the predicted spectral index of the radio emission is different to the observed spectral index.

Acknowledgements. We thank G. C. Dewangan, B. Paul, M. Choudhury, and J. S. Yadav for very useful discussions. This research has made use of data obtained through the High Energy Astrophysics Science Archive Research Center Online Service, provided by the NASA/Goddard Space Flight Center. The Green Bank Interferometer is a facility of the National Science Foundation operated by the NRAO in support of NASA High Energy Astrophysics programs.

\section{Note added in proof}

It was pointed out to us that the total radiated power from the jet (by integrating the predicted spectra in Fig. 4) comes more than the Eddington luminosity of this source $\left(\sim 10^{39} \mathrm{erg} \mathrm{s}^{-1}\right)$. Such a high radiated power from the jet is unlikely. However, this is because of the rather steep spectral index in the optically thick region which causes a high flux at the turnover frequency. The observed flux in the radio band is $\sim 10^{31} \mathrm{erg} \mathrm{s}^{-1}$ and the observed power in $\mathrm{X}$-rays due to the jet (as suggested here) is $\sim 10^{38} \mathrm{erg} \mathrm{s}^{-1}$. Hence the high radiated power in the jet is mainly due to the large peak at $\sim 10^{14} \mathrm{~Hz}$. A more realistic model which directly connects radio to X-rays by (a) incorporating models which predict flatter spectral index in the radio band (Falcke 1996; Falcke \& Biermann 1999) and (b) a model incorporating energy loss which will have one more spectral turn over at intermediate frequencies, might be able to give more realistic value for radiated jet power. It should also be noted that Gliozzi et al. (1999; MNRAS
303, L37) have estimated a power in kinetic bulk motion of GRS $1915+105$, which far exceeds the Eddington luminosity.

\section{References}

Belloni, T., Mendez, M., King, A. R., van der Klis, M., \& van Paradijs, J. 1997, ApJ, 488, L109

Belloni, T., Klein-Wolt, M., Mendez, M., van der Klis, M., \& van Paradijs, J. 2000, A\&A, 355, 271

Blandford, R., \& Konigl, A. 1979, ApJ, 23234

Castro-Tirado, A. J., Brandt, S., \& Lund, N. 1992, IAU Circ., 5590

Chakrabarti, S. K. 1999, A\&A, 351, 185

Chakrabarti, S. K., \& Titarchuk, L. G. 1995, ApJ, 455, 623

Chen, X., Swank, J. H., \& Taam, R. E. 1997, ApJ, 477, L41

Das, T. K., \& Chakrabarti, S. K. 1999, Classical and Quantum Gravity, 16, 19, 3879

Dhawan, V., Mirabel, I. F., \& Rodriguez, L. F. 2000, ApJ, 543, 373

Falcke, H. 1996, ApJ, 464, L67

Falcke, H., \& Biermann, P. L. 1996, A\&A, 308, 321

Falcke, H., \& Biermann, P. L. 1999, A\&A, 342, 49

Fender, R. P., Garrington, S. T., McKay, D. J., et al. 1999, MNRAS, 304, 865

Gierlinski, M., Zdziarski, A. A., Poutanen, J., et al. 1999, MNRAS, 309, 496

Hjellming, R. M., \& Johnston, K. J. 1988, ApJ, 328, 600

Jahoda, K., et al. 1996, SPIE, 2808, 59

Kotani, T., Ebisawa, K., Dotani, T., Inoue, H., et al. 2000, ApJ, 539, 413

Lee, J. C., Schulz, N. S., Reynolds, C. S., Fabian, A. C., \& Blackman, E. G. 2000, Contributed talk at "X-ray Astronomy 2000", Palermo, Italy, Sept. 2000 [astro-ph/0012111]

Longair, M. S. 1994, High Energy Astrophysics, Vol. 2, 2nd edition, CUP

Markoff, S., Falcke, H., \& Fender, R. 2000, accepted in A\&AL [astro-ph/0010560]

Markwardt, C. B., Swank, J. H., \& Taam, R. E. 1999, ApJ, 513, L37

Mirabel, I. F., \& Rodriguez, L. F. 1994, Nature, 371, 46

Mirabel, I. F., \& Rodriguez, L. F. 1999, ARA\&A, 37, 409

Morgan, E. H., Remillard, R. A., \& Greiner, J. 1997, ApJ, 482, 993

Muno, M. P., Morgan, E. H., \& Remillard, R. A. 1999, ApJ, 527,321

Naik, S., \& Rao, A. R. 2000, A\&A, 362, 691

Narayan, R., Mahadevan, R., \& Quataert, E. 1998, The Theory of Black Hole Accretion Disks, CUP

Rao, A. R., Naik, S., Vadawale, S. V., \& Chakrabarti, S. K. 2000, A\&A, 360, L25

Rao, A. R., Yadav, J. S., \& Paul, B. 2000, ApJ, 544, 443

Rao, A. R., \& Vadawale, S. V. 2000, Presented at the 4th Integral Workshop, Alicante, Sept. 4-8, 2000

Reynolds, S. P. 1982, ApJ, 256, 13

Rothschild, R. E., et al. 1998, ApJ, 496, 538

Sunyaev, E. A., \& Titarchuk, L. 1980, A\&A, 86, 121

Trudolyubov, S., Churazov, E., \& Gilfanov, M. 1999, Ast. L., 25,718

Yadav, J. S., Rao, A. R., Agrawal, P. C., et al. 1999, ApJ, 517, 935 